\section{BMJ Paediatrics Open}

\title{
Health-related quality of life after camp-based family obesity treatment: an RCT
}

\author{
Beate Benestad, ${ }^{\oplus 1,2}$ Tor-Ivar Karlsen, ${ }^{3}$ Milada Cvancarova Småstuen, ${ }^{1}$ \\ Samira Lekhal, ${ }^{1}$ Jens Kristoffer Hertel, ${ }^{1}$ Silje Steinsbekk, ${ }^{4,5}$ Ronette L Kolotkin, ${ }^{1,6}$ \\ Rønnaug Astri Ødegård, ${ }^{7,8}$ Jøran Hjelmesæth ${ }^{1,9}$
}

To cite: Benestad B, Karlsen T-I, Småstuen MC, et al. Health-related quality of life after camp-based family obesity treatment: an RCT. BMJ Paediatrics Open 2019:3:e000413. doi:10.1136/ bmjpo-2018-000413

- Prepublication history and additional material for this paper are available online. To view these files, please visit the journal online (http://dx.doi.org/ 10.1136/bmjpo-2018-000413).

Received 3 December 2018 Revised 19 February 2019 Accepted 26 February 2019
Check for updates

(C) Author(s) (or their employer(s)) 2019. Re-use permitted under CC BY-NC. No commercial re-use. See rights and permissions. Published by BMJ.

For numbered affiliations see end of article.

Correspondence to Dr Beate Benestad; beate. benestad@siv.no

\section{ABSTRACT}

Objective To compare the effects of a 2-year campbased immersion family treatment for obesity with an outpatient family-based treatment for obesity on healthrelated quality of life (HRQoL) in two generations.

Design Randomised controlled trial.

Setting Rehabilitation clinic, tertiary care hospital and primary care.

Patients Families with at least one child (7-12 years) and one parent, both with obesity.

Interventions Summer camp for 2 weeks, with four repetition weekends, or lifestyle school, including four outpatient days over 4 weeks. Behavioural techniques to promote a healthier lifestyle.

Main outcome measures Children's and parents' HRQoL were assessed using generic and obesity-specific measures. Outcomes were analysed using linear mixed models according to intention to treat, and multiple imputations were used for missing data.

Results Ninety children (50\% girls) with a mean (SD) age of 9.7 (1.2) years and body mass index $28.7(3.9) \mathrm{kg} /$ $\mathrm{m}^{2}$ were included in the analyses. Summer camp children had an estimated mean $(95 \% \mathrm{Cl})$ of $5.3(0.4$ to 10.1$)$ points greater improvement in adiposity-specific HRQoL score at 2 years compared with the lifestyle school children, and this improvement was even larger in the parent proxyreport, where mean difference was $7.3(95 \% \mathrm{Cl} 2.3$ to 12.2). Corresponding effect sizes were 0.33 and 0.44 . Generic HRQoL questionnaires revealed no significant differences between treatment groups in either children or parents from baseline to 2 years.

Conclusions A 2-year family camp-based immersion obesity treatment programme had significantly larger effects on obesity-specific HRQoL in children's selfreport and parent proxy-reports in children with obesity compared with an outpatient family-based treatment programme.

Trial registration number NCT01110096.

\section{INTRODUCTION}

Both psychological and physical health are negatively affected by obesity. ${ }^{12}$ Accordingly, childhood obesity treatment also aims to improve psychosocial well-being and health-related quality of life (HRQoL). ${ }^{3}$ However, there is conflicting evidence regarding the effect

\section{What is already known on this topic?}

Children and adults with obesity often have reduced generic and obesity-specific health-related quality of life (HRQoL) compared with normal-weight peers.

- Both children and adults report improvement in HRQoL after intensive lifestyle treatment for obesity.

- Previous camp-based studies of HRQoL in children treated for obesity were not randomised, only one included a control group and none reported parents HRQLL.

\section{What this study hopes to add?}

Summer camp children had larger improvements in obesity-specific HRQoL compared with lifestyle school children, despite no significant differences in body mass index SD score at the end of treatment.

- There were no statistically significant differences in HRQoL between parents in the camp-based programme and parents in the outpatient treatment programme at 2 years.

of non-surgical multidisciplinary weight loss intervention programmes on HRQoL. ${ }^{3}{ }^{4}$ This might be due to the fact that most treatments only yielded no or modest weight loss. ${ }^{5}$ Furthermore, although camp-based treatment of childhood obesity has shown promising results, ${ }^{6}$ only a few non-randomised studies included HRQoL. ${ }^{7-9}$

It has been suggested that targeting both parent and child may enhance treatment effectiveness compared with child-only interventions. ${ }^{1011}$ To our knowledge, no study has assessed the effect on HRQoL of including both child and parent with obesity in the camp-based treatment.

Previously, we reported results of a 2-year randomised controlled trial (RCT) of a family summer camp treatment compared with an outpatient lifestyle intervention, finding no 
significant between-group differences after 2 years for primary outcomes (age-adjusted and sex-adjusted body mass index [BMI] SD score [BMI-SDS] for children's and parents' BMI) ${ }^{12}$ However, these treatment options may still affect HRQoL differently.

The aim of the present study was therefore to assess the effect of the interventions on HRQoL, a prespecified secondary outcome. We hypothesised greater improvements in both general and obesity-specific HRQoL in both children and parents undergoing summer camp treatment versus those receiving outpatient lifestyle treatment.

\section{PATIENTS AND METHODS}

\section{Study design and setting}

The Family based intervention in childhood obesitY was a pragmatic two-armed RCT conducted at two tertiary care centres in Norway. Data collection occurred between April 2010 and June 2013. Details of the trial have been published previously. ${ }^{12}$ Patients were not directly involved in the design of this study.

\section{Participants}

Families with at least one child with obesity (International Obesity Task Force), ${ }^{13}$ aged $7-12$ years and at least one parent with obesity (BMI $\geq 30 \mathrm{~m} / \mathrm{kg}^{2}$ ) were recruited from primary healthcare nurses and general practitioners $(>75 \%)$, as well as from media and regular referrals in $2010 \quad(\mathrm{n}=39)$ and $2011 \quad(\mathrm{n}=55)$. Exclusion criteria were syndromal obesity, other medical conditions associated with weight gain or inability to participate in either of the treatment programmes. Written informed consent was provided from all participants, and the study was performed in accordance with the Declaration of Helsinki.

\section{Interventions and outcomes}

Summer camp participants underwent an initial 2-week programme at a private rehabilitation institution with four follow-up weekends (2 days at 6, 12, 18 and 24 months). The lifestyle school group attended 4 days (23 hours) in the outpatient clinic over a period of 4 weeks. All participants were offered monthly primary care follow-up for 2 years by a public health nurse. All interventions focused on healthy choices in terms of nutrition and physical activity and were based on behavioural techniques. ${ }^{14-16}$ Details of both treatment programmes have been described previously. ${ }^{12}$

At baseline, demographic information was obtained in semistructured interviews, and clinical examinations were performed. Anthropometric characteristics were recorded at baseline, at 1 year (only children) and at 2 years. HRQoL questionnaires were completed at baseline and at 2 years.

\section{HRQOL measures}

Age-specific self-report and parent proxy versions of the 'KINDer Lebensqualitätsfragebogen' (KINDL) questionnaire $^{1718}$ and its obesity-specific module ${ }^{19}$ were completed by children and parents. The KINDL is a 24-item generic HRQoL instrument representing six dimensions of HRQoL (physical well-being, emotional well-being, self-esteem, family, friends and school). A total score and subscale scores were calculated. The KINDL obesity-specific disease module contains 15 items, 12 of which are used to calculate an adiposity scale. All scales are transformed into ranges from 0 to 100 ; higher scores indicate better HRQoL. The KINDL is a reliable and valid instrument for measuring HRQoL. ${ }^{18}$ The KINDL adiposity scale has a Cronbach's alpha of $0.77 .^{20}$

Parents completed two adult, self-report obesity-specific questionnaires, Obesity and Weight Loss Quality of Life ${ }^{21} 22$ and Weight Related Symptom Measure (WRSM), ${ }^{21} 22$ plus the generic Short-Form 36-item Health Survey (SF-36) ${ }^{23}$ at baseline and the 2-year visit. The 17-item OWLQOL measures behaviours and feelings that are associated with overweight/obesity and weight loss. A score of 0 indicates the greatest adverse impact, and a score of 100 indicates the lowest impact, thus increasing OWLQOL scores imply better HRQoL. The WRSM is a 20 -item instrument assessing the presence and bothersomeness of symptoms. Scores range from 0 to 120 , with higher scores indicating a higher symptom burden. The SF-36 measures an individual's general health status across eight subscales (physical functioning, role physical, bodily pain, general health, vitality, social functioning, role emotional and mental health). Two summary measures-the physical component summary and the mental component summary-are calculated from the eight scales using different weightings. Lower scores represent more impaired health status, with a score of 50 being the mean for the US general population. Results are presented as correlated oblique physical and mental health factor summary scores. ${ }^{24}$ The scores were transformed in accordance with the specific instructions from the authors of the different questionnaires.

\section{Sample size and randomisation}

The sample size was calculated based on the primary outcome measure (BMI SDS) for the children. ${ }^{12}$ After inclusion of each consecutive family, study personnel contacted technical staff at the randomisation centre. Block randomisation (block sizes of four and five participants) stratified by the treatment centre was computer generated by technical staff using an internet-based device. Randomisation was performed 2 days after the baseline measurements. The participants (families) were randomly assigned to one of the two parallel groups in a 1:1 ratio. Allocation was concealed from both participants and triallists. Blinding of study participants or healthcare professionals was not possible due to the nature of the interventions.

\section{Statistical analyses}

Baseline data are presented as means and SDs or counts (percentages). Crude differences between pairs 
of continuous and categorical variables were assessed using independent samples t-test, Mann-Whitney U test, Wilcoxon signed rank test or Fisher's exact test as appropriate.

All individuals were analysed according to the intention-to-treat principle. To account for repeated measures on the same individuals, data were analysed using a linear mixed model, ${ }^{25}$ with an unstructured covariance matrix. Fixed effects were treatment group, time and time*treatment group interaction. After applying Little's test of randomness of missing data $(\mathrm{p}=0.909)$, missing values on the KINDL (children and parents) and adult HRQoL (parents) scales at baseline and 2 years were imputed using multiple imputation. The multiple imputation was performed using a fully conditional specification model, applying linear regression as the prediction method for scale variables and two-way interactions for categorical variables. We generated five complete datasets with 10 iterations per dataset. Statistical analyses were performed on each imputed dataset, and thereafter the results were combined to arrive to single estimates. The combined estimates are presented.

We estimated mean changes in HRQoL from baseline to 2 years, and results are presented as estimated mean difference between the treatment groups with 95\% CIs. The standardised Cohen's d (effect sizes [ESs]) of the outcomes were calculated. ${ }^{26}$ In addition, to test the robustness of the results, we performed a sensitivity analysis, using the same linear mixed model with all available data but without imputation of missing variables. We tested reliability of the sub- and total scales of the questionnaires calculating Cronbach's alpha coefficient.

All tests were two sided. $\mathrm{P}$ values $<0.05$ were considered statistically significant. All analyses were performed with SPSS V.25.0.

\section{RESULTS}

Participant flow is depicted in figure 1. Table 1 shows baseline demographic and clinical characteristics of the 90 children and 89 parents that were available for the intention-to-treat analysis. ${ }^{12}$ Online supplementary table 1 shows the numbers of valid questionnaires for each time point.

Values are reported as mean (SD) or number (\%). Statistics were independent samples t-test for normally distributed continuous data, Mann-Whitney $U$ test for non-normally distributed continuous data or Fisher's exact test for categorical data.

With a few exceptions, baseline demographics, clinical characteristics and HRQoL scores did not differ significantly between the groups (tables 1 and 2). There was, however, a higher percentage of female parents in the lifestyle school group ( $88 \%$ vs $76 \%$ ) than in the summer camp group (table 1) and a small imbalance in the school domain of the parent proxy-report version of KINDL (table 2).

\section{Changes in HRQOL}

Compared with the lifestyle school group, the summer camp group showed significantly greater improvement from baseline to 2 years on the KINDL adiposity module scores, both when self-reported and parent proxy reported (table 2, figure 2). Children's self-report and parent proxy-report revealed estimated mean differences $(95 \%$ CIs) in the obesity-specific scores of 5.3 ( 0.4 to 10.1 ) points and 7.3 (2.3 to 12.2), respectively. Corresponding ESs were 0.33 and 0.44. A sensitivity analysis using the same linear mixed model without replacing missing values showed similar results (supplementary table 2).

Generic HRQoL measures did not reveal any significant differences between the two treatment groups regarding changes from baseline to 2 years for either children or parents (table 2, figure 2), neither did the obesity-specific HRQoL measures for the parents.

Considering the fraction of missing information, relative increase in variance and relative efficiency, the imputed data-sets were comparable with the original data-set (data not shown).

All scales in the parent proxy version of KINDL had satisfactory reliability (Cronbach's alpha values $>0.70$ ), except for the subscales of physical well-being and school. The children's version of KINDL had satisfactory total score reliability, but all subscales had Cronbach's alpha values $<0.70$. The child and parent proxy version of the KINDL adiposity-specific module and all adult HRQoL questionnaires had Cronbach's alpha values $>0.80$ (online supplementary table 3 .)

\section{DISCUSSION}

This RCT of families affected by obesity compared the 2-year effect of a family camp-based treatment programme (summer camp) with the effect of an outpatient treatment programme (lifestyle school) on HRQoL. This is the first RCT to explore the long-term effects of a campbased intervention in both children and parents. Children receiving camp-based treatment had significantly greater improvements in the obesity-specific measures of HRQoL, both using self-report and parent proxy, than children in the lifestyle school group. Corresponding ESs were small to medium. In contrast, generic HRQoL measures showed no significant between-group differences from baseline to 2 years for either children or parents, nor did the obesity-specific HRQoL measures for the parents. The finding of between-group differences on obesity-specific HRQoL, but not on generic measures of HRQoL, is not unusual. Disease-specific measures of HRQoL are generally more responsive to intervention than generic measures. ${ }^{27}$

It is noteworthy that 2-year improvements in obesity-specific HRQoL were demonstrated for summer camp participants compared with lifestyle-school participants, both on self-report and parent proxy-report, despite there being no differences in BMI-SDS. ${ }^{12}$ This 


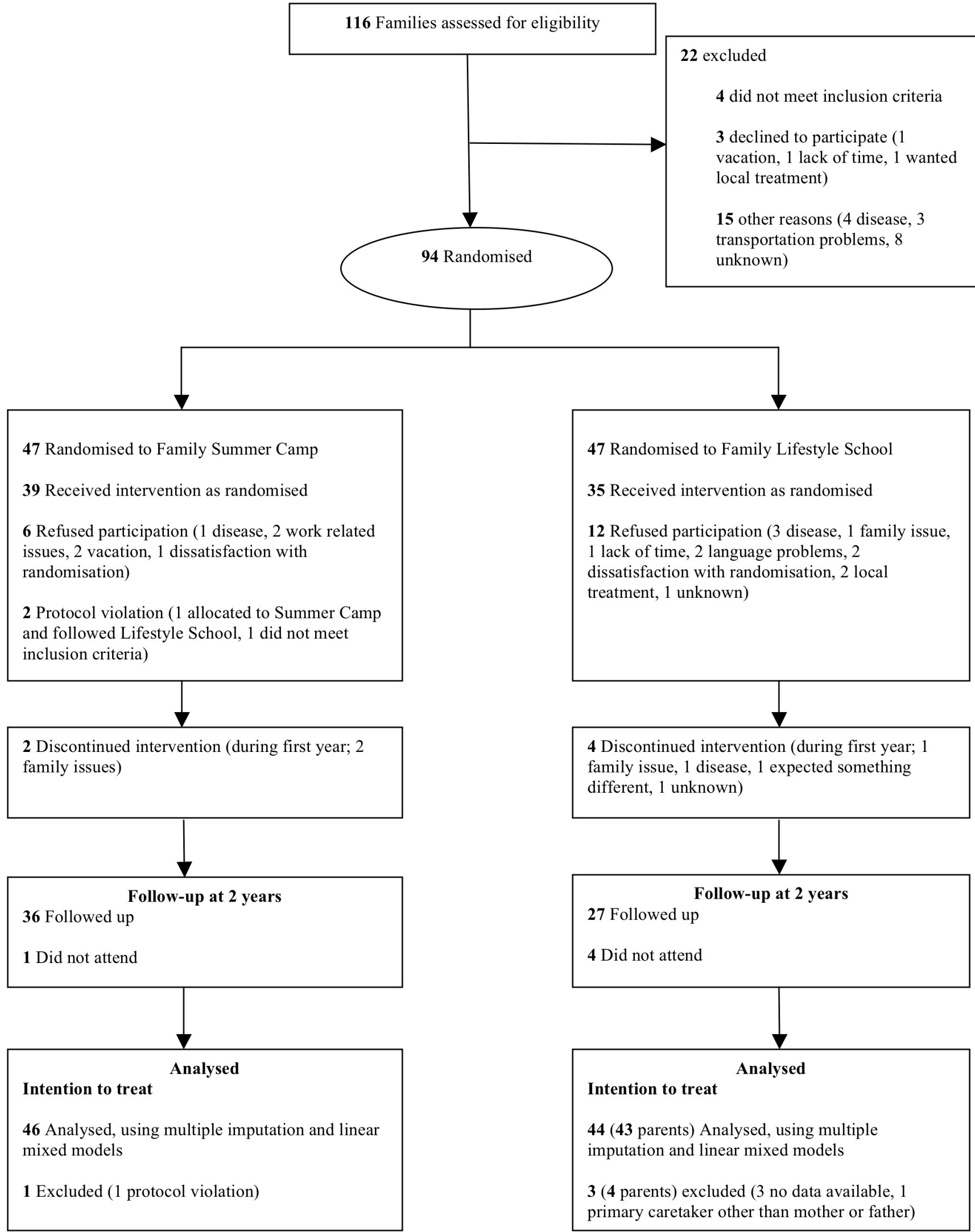

Figure 1 Participant flow: families with obesity assessed for eligibility, randomisation, intervention and follow-up.

is encouraging, as it suggests that the feelings, physical challenges and complaints associated with obesity may improve after comprehensive treatment for obesity even in the absence of significant weight loss. Given that poor self-image, bullying and bodily pain are prevalent in youth with obesity in clinical populations, ${ }^{1}$ any change 
Table 1 Baseline demographic and clinical characteristics of the 90 children and 89 parents included in the analyses

\begin{tabular}{|c|c|c|c|}
\hline & All & $\begin{array}{l}\text { Family } \\
\text { summer } \\
\text { camp }\end{array}$ & $\begin{array}{l}\text { Family } \\
\text { lifestyle } \\
\text { school }\end{array}$ \\
\hline Children & $\mathrm{n}=90$ & $n=46$ & $n=44$ \\
\hline $\begin{array}{l}\text { Gender, female } \\
(\%)\end{array}$ & $45(50)$ & $20(44)$ & $25(57)$ \\
\hline $\begin{array}{l}\text { Ethnicity, } \\
\text { European white } \\
\text { (\%) }\end{array}$ & $77(86)$ & 41 (89) & $36(82)$ \\
\hline Age (years) & 9.7 (1.2) & $9.6(1.1)$ & 9.7 (1.2) \\
\hline $\mathrm{BMI}\left(\mathrm{kg} / \mathrm{m}^{2}\right)$ & 28.7 (3.9) & $28.2(4.1)$ & $29.3(3.7)$ \\
\hline BMI SDS & $3.46(0.75)$ & $3.41(0.79)$ & $3.51(0.71)$ \\
\hline Parents & $\mathrm{n}=89$ & $\mathrm{n}=46$ & $n=43$ \\
\hline $\begin{array}{l}\text { Gender, female } \\
(\%)\end{array}$ & $69(78)$ & $31(67)$ & 38 (88) \\
\hline Age (years) & $40.7(5.0)$ & $40.9(4.8)$ & $40.6(5.2)$ \\
\hline BMI $\left(\mathrm{kg} / \mathrm{m}^{2}\right)$ & $37.0(4.6)$ & $37.0(4.4)$ & 36.9 (4.9) \\
\hline
\end{tabular}

BMI SDS; body mass index SD score.

in HRQoL is likely to be perceived as a recognisable improvement for these children. Scores between 4 and 5 points on a scale from 0 to 100 have been considered to be minimal clinically meaningful differences previously. ${ }^{4}$ Our results are in accordance with previous studies that have reported improved psychosocial functioning even after controlling for BMI change. For example, Quinlan et $a l^{8}$ found improved social functioning, physical functioning, weight and eating efficacy, after controlling for BMI change in a camp-based treatment programme.

Camp-based treatment can be considered to be 'immersion treatment', which has been described as treatment in a 'therapeutic and educational environment for extended periods of time, thereby removing participants from obesogenic environments' ${ }^{6}{ }^{6}$ In the present study, children in the camp-based group were immersed in a non-obesogenic environment, initially for 2 weeks, followed by four follow-up weekends. Early success achieved through immersion treatment may improve self-efficacy, attitudes and moods, which in turn may enhance long-term commitment. ${ }^{6}$ In addition, the children participating in the summer camp group might have experienced an increase in social support from their participating peers ${ }^{28}$ which could have led to higher self-acceptance and increased HRQoL. ${ }^{29}$ This in turn could have affected both their motivation for treatment and their self-efficacy. ${ }^{28}$

Comparing our results directly with other camp-based immersion treatment studies proved challenging, as the few existing studies used different questionnaires for measuring HRQoL. Although previous camp-based studies did not include parents together with their children, Knöpfli et $a l^{7}$ gave parents practical and theoretical counselling in their multidisciplinary inpatient programme, but they only focused on treatment of the children's obesity. They reported increased HRQoL for the children pretreatment to post-treatment over 8 weeks, as well as a large effect on weight. Nonetheless, they did not include a control group or a subsequent follow-up. A study of a combined inpatient (6 weeks) and outpatient (4.5 months) treatment programme included a waiting list control group, and it found an increase in obesity-specific HRQoL ${ }^{30}$ in the treatment group compared with the control group. ${ }^{9}$ Improvements in both generic and weight-related quality of life immediately after a summer camp weight loss programme have been demonstrated for adolescents ${ }^{8}$ and children, ${ }^{31}$ although these studies did not include a control group or longer follow-up. A more recent observational study demonstrated improvements in both generic and weight-related HRQoL among children and adolescents (8-19 years) with severe obesity undergoing an intensive lifestyle treatment for 1 year despite partial weight regain from 1 -year to 2-year measurements. The treatment included an inpatient period of either 2 or 6 months (immersion treatment) and then a 1-year follow-up. ${ }^{32}$ This study also found improvements in self-reported obesity-specific HRQoL comparable with the current study, of 8.9 (4.6 to 13.2) points, although using a different HRQoL instrument ${ }^{33}$ (with scores ranging from 0 to 100 , as in the KINDL). In the present study, the improvements in obesity-specific HRQoL scores were not accompanied by improvements in BMI SDS. This is in accordance with results from another study with 24-month follow-up after 4-6 weeks of inpatient treatment, which did not find an association between changes in HRQoL and changes in BMI but indicated a potential role of physical activity in improving HRQoL. ${ }^{34}$

\section{Strengths and limitations}

The relatively long follow-up is a strength in this study. Our findings might be generalisable to treatment-seeking families with obesity and applicable in similar healthcare settings engaged in childhood obesity treatment. In addition, we assessed HRQoL in both children and parents participating in the study and applied both self-reports and parent proxy-reports for the children. The use of disease-specific, in addition to generic, instruments to measure HRQoL is an advantage, as they are more responsive to effects of treatment. ${ }^{27}$

Limitations include the participation of mainly European white families and the lack of data on socioeconomic status and adherence to follow-up in the municipalities. Furthermore, neither of the interventions in this study represent the standard care treatment at the tertiary care centres. Weight inclusion criteria for the study was lower than criteria for specialist treatment. The sample size was calculated based on the primary outcome measure (BMI SDS) for the children, not on the HRQoL measures. We did not include measures of motivation or perceived social support. Another limitation is that reliability of the KINDL subscales for the children's version (all subscales) 


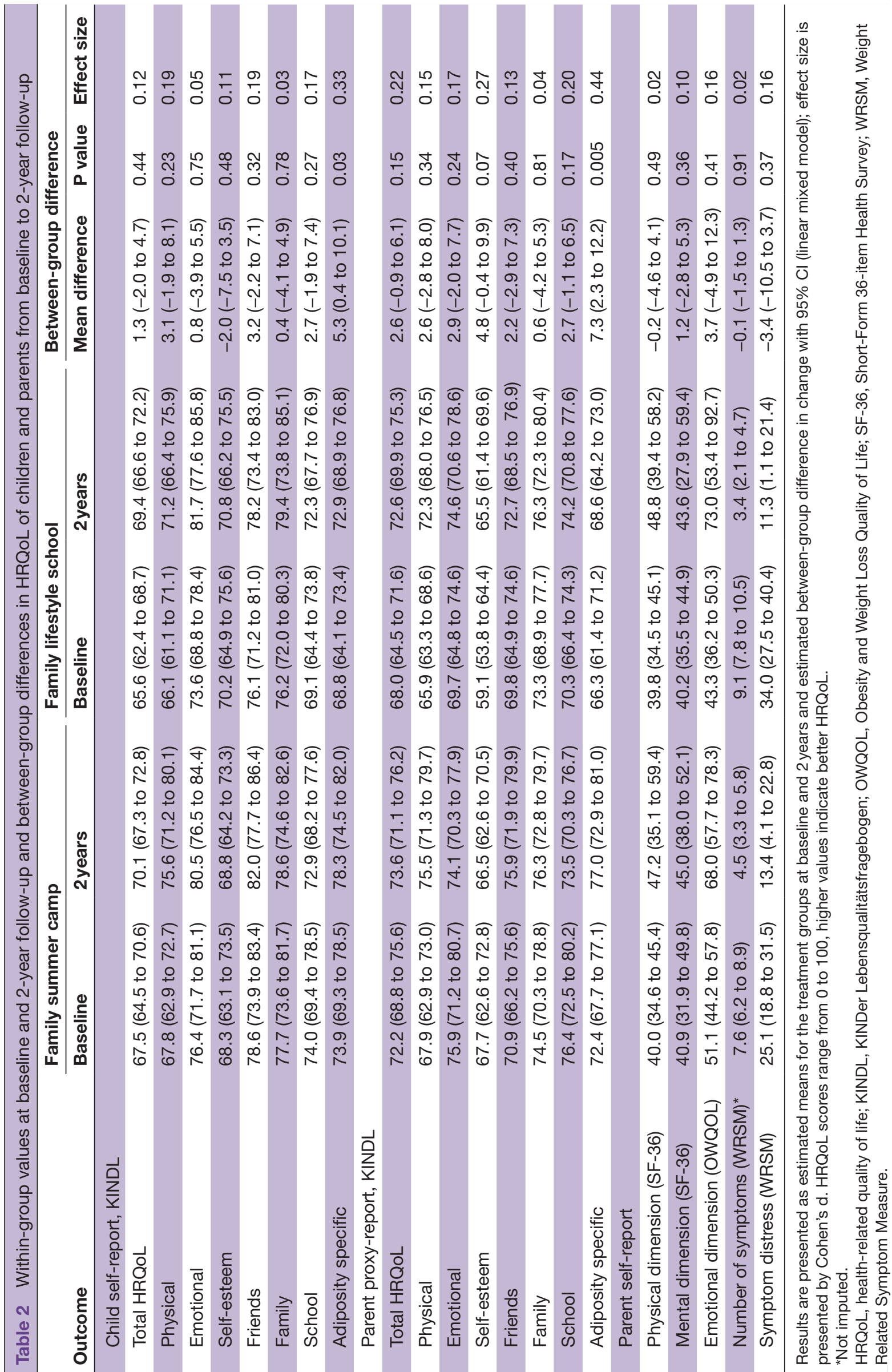




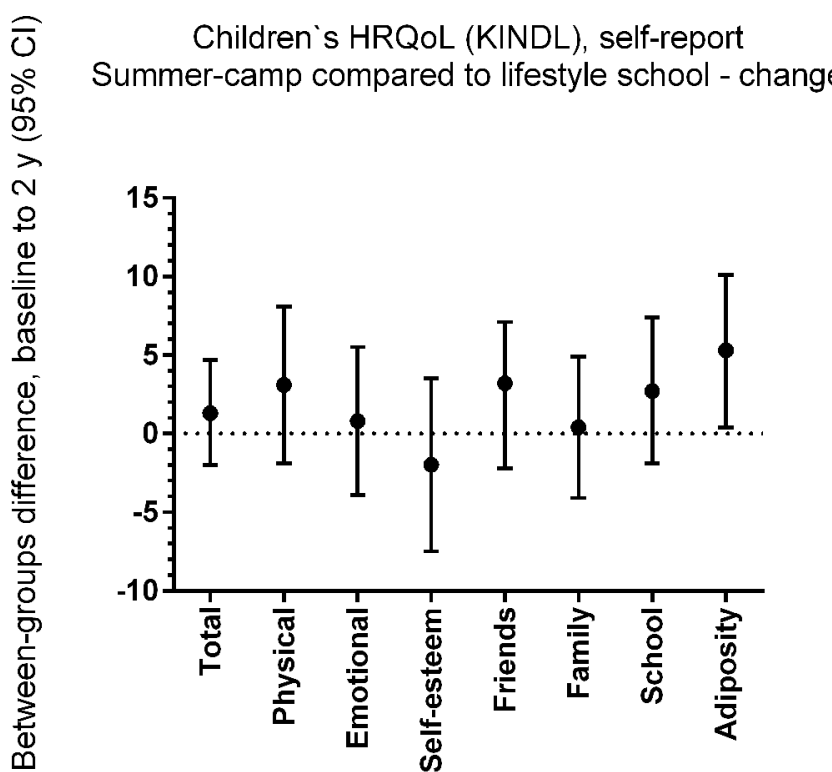

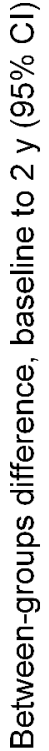

Children's HRQoL (KINDL), parent proxy-report Summer-camp compared to lifestyle school - change

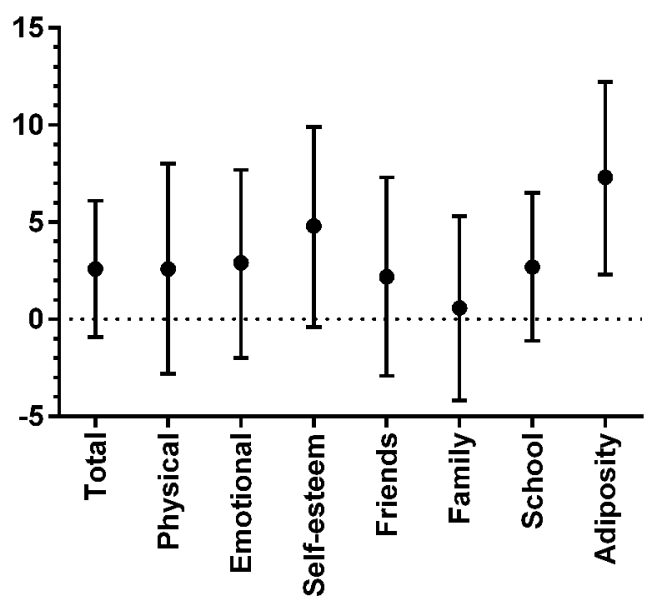

Figure 2 Estimated mean difference in change betweengroups $(95 \% \mathrm{Cl})$ in HRQoL scores for the children from baseline to 2 years, measured by KINDL self-reports (upper) and parent proxy-reports (bottom). The possible scale for $\mathrm{HRQ}$ oL range from 0 to 100 . HRQoL, health-related quality of life; KINDL, KINDer Lebensqualitätsfragebogen.

and some of the parent's proxy reports (physical wellbeing and school) were unsatisfactory. Similar values for reliability of the subscales of the KINDL have previously been shown by others. ${ }^{29} 35$

\section{CONCLUSION}

A 2-year family camp-based immersion treatment programme for obesity resulted in significantly larger improvements in obesity-related HRQoL in children's self-reports and parent proxy-reports in children with obesity compared with an outpatient family-based treatment programme despite no significant effects on BMI SDS.

Author affiliations

${ }^{1}$ Department of Medicine, Vestfold Hospital Trust, The Morbid Obesity Centre (MOC), Tønsberg, Norway

${ }^{2}$ Faculty of Medicine, University of Oslo, 0slo, Norway

${ }^{3}$ Faculty of Health and Sports Sciences, University of Agder, Grimstad, Norway

${ }^{4}$ Department of Psychology, Norwegian University of Science and Technology,

Trondheim, Norway

${ }^{5}$ Department of Social Science, Norwegian University of Science and Technology, Trondheim, Norway

${ }^{6}$ Quality of Life Consulting, Durham, North Carolina, USA

${ }^{7}$ The Obesity Centre, St. Olavs Hospital, Trondheim University Hospital, Trondheim, Norway

${ }^{8}$ Department of Clinical and Molecular Medicine, Norwegian University of Science and Technology, Trondheim, Norway

${ }^{9}$ Department of Endocrinology, Morbid Obesity and Preventive Medicine, Institute of Clinical Medicine, University of Oslo, 0slo, Norway

Acknowledgements We are grateful for the contributions of all healthcare professionals involved at the four centres and all the children and parents who participated in this study. We particularly wish to thank the former leader of the MOC pediatric section, MD Martin Handeland, study nurse Åshild Skulstad-Hansen and bioengineer Berit Mossing Bjørkås. Research relating to this article was funded by the Norwegian Ministry of Health and Care Services, the Norwegian Foundation for Health and Rehabilitation and GjensidigeStiftelsen. The first author has been funded by a public research grant from the South-Eastern Norway Regional Health Authority. Treatment in the Norwegian public healthcare system is nearly free of charge for patients.

Contributors BB carried out the analyses, drafted the initial manuscript, reviewed and revised the manuscript and approved the final manuscript as submitted. T-IK designed parts of the study protocol, carried out the multiple imputations, gave advice on the initial manuscript, reviewed and revised the manuscript and approved the final manuscript as submitted. MCS gave advice on the statistical analyses, reviewed and revised the manuscript and approved the final manuscript as submitted. SL coordinated and supervised parts of the data collection at one of the outpatient clinics, reviewed and revised the manuscript and approved the final manuscript as submitted. JKH gave advice on data preparation, contributed to discussion, reviewed and revised the manuscript and approved the final manuscript as submitted. SS contributed to discussion, reviewed and revised the manuscript and approved the final manuscript as submitted. RLK contributed to discussion, reviewed and revised the manuscript and approved the final manuscript as submitted. RA $\emptyset$ designed the study and wrote the protocol, coordinated and supervised data collection at one of the outpatient clinics, reviewed and revised the manuscript and approved the final manuscript as submitted. JH designed the study and wrote the protocol, reviewed the initial manuscript, reviewed and revised the manuscript and approved the final manuscript as submitted. All authors approved the final manuscript as submitted and are accountable for all aspects of the work.

Funding Research relating to this article was funded by the Norwegian Ministry of Health and Care Services, the Norwegian Foundation for Health and Rehabilitation and GjensidigeStiftelsen. The first author has been funded by a public research grant from the South-Eastern Norway Regional Health Authority.

Disclaimer None of the funding parties had a role in design or conduct of the study; collection, management, analysis or interpretation of the data; or preparation, review or approval of the manuscript.

Competing interests None declared.

Patient consent for publication Not required.

Ethics approval The study was approved by the Regional Committee for Medical and Health Research Ethics (2009/176).

Provenance and peer review Not commissioned; externally peer reviewed.

Open access This is an open access article distributed in accordance with the Creative Commons Attribution Non Commercial (CC BY-NC 4.0) license, which permits others to distribute, remix, adapt, build upon this work non-commercially, and license their derivative works on different terms, provided the original work is properly cited, appropriate credit is given, any changes made 
indicated, and the use is non-commercial. See: http://creativecommons.org/ licenses/by-nc/4.0/.

\section{REFERENCES}

1. Buttitta M, Iliescu C, Rousseau A, et al. Quality of life in overweight and obese children and adolescents: a literature review. Qual Life Res 2014;23:1117-39.

2. Halfon N, Larson K, Slusser W. Associations between obesity and comorbid mental health, developmental, and physical health conditions in a nationally representative sample of US children aged 10 to 17. Acad Pediatr 2013;13:6-13.

3. Steele RG, Gayes LA, Dalton WT, et al. Change in health-related quality of life in the context of pediatric obesity interventions: A meta-analytic review. Health Psychol 2016;35:1097-109.

4. Ligthart KA, Paulis WD, Djasmo D, et al. Effect of multidisciplinary interventions on quality of life in obese children: a systematic review and meta-analysis. Qual Life Res 2015;24:1635-43.

5. Janicke DM, Steele RG, Gayes LA, et al. Systematic review and meta-analysis of comprehensive behavioral family lifestyle interventions addressing pediatric obesity. J Pediatr Psychol 2014;39:809-25

6. Kelly KP, Kirschenbaum DS. Immersion treatment of childhood and adolescent obesity: the first review of a promising intervention. Obes Rev 2011;12:37-49.

7. Knöpfli BH, Radtke T, Lehmann M, et al. Effects of a multidisciplinary inpatient intervention on body composition, aerobic fitness, and quality of life in severely obese girls and boys. $J$ Adolesc Health 2008;42:119-27.

8. Quinlan NP, Kolotkin RL, Fuemmeler BF, et al. Psychosocial outcomes in a weight loss camp for overweight youth. Int $\mathrm{J}$ Pediatr Obes 2009:4:134-42.

9. Adam S, Westenhofer J, Rudolphi B, et al. Effects of a combined inpatient-outpatient treatment of obese children and adolescents. Obes Facts 2009;2:286-93.

10. Epstein LH, Valoski A, Wing RR, et al. Ten-year follow-up of behavioral, family-based treatment for obese children. JAMA 1990;264:2519-23.

11. Wrotniak BH, Epstein LH, Paluch RA, et al. Parent weight change as a predictor of child weight change in family-based behavioral obesity treatment. Arch Pediatr Adolesc Med 2004;158:342-7.

12. Benestad B, Lekhal S, Småstuen MC, et al. Camp-based family treatment of childhood obesity: randomised controlled trial. Arch Dis Child 2017;102:303-10.

13. Cole TJ, Bellizzi MC, Flegal KM, et al. Establishing a standard definition for child overweight and obesity worldwide: international survey. BMJ 2000;320:1240-3.

14. Poey K. Guidelines for the practice of brief, dynamic group therapy. Int J Group Psychother 1985;35:331-54.

15. Rollnick S, Kinnersley P, Stott N. Methods of helping patients with behaviour change. BMJ 1993;307:188-90.

16. Ogden T, Hagen KA. Treatment effectiveness of parent management training in Norway: a randomized controlled trial of children with conduct problems. J Consult Clin Psychol 2008;76:607-21.

17. Ravens-Sieberer U, Bullinger M. Assessing health-related quality of life in chronically ill children with the German KINDL: first psychometric and content analytical results. Qual Life Res 1998;7:399-407.

18. Bullinger M, Brütt AL, Erhart M, et al. Psychometric properties of the KINDL-R questionnaire: results of the BELLA study. Eur Child Adolesc Psychiatry 2008;17(Suppl 1):125-32.

19. Bullinger M, Ravens-Sieberer U. The KINDL questionnaires. https:// www.kindl.org/english/questionnaires/

20. Wille N, Bullinger M, Holl R, et al. Health-related quality of life in overweight and obese youths: results of a multicenter study. Health Qual Life Outcomes 2010;8:36.

21. Niero M, Martin M, Finger T, et al. A new approach to multicultural item generation in the development of two obesity-specific measures: the Obesity and Weight Loss Quality of Life (OWLQOL) questionnaire and the Weight-Related Symptom Measure (WRSM). Clin Ther 2002;24:690-700.

22. Patrick DL, Bushnell DM, Rothman M. Performance of two selfreport measures for evaluating obesity and weight loss. Obes Res 2004;12:48-57.

23. Ware JE, Sherbourne CD. The MOS 36-item short-form health survey (SF-36). I. Conceptual framework and item selection. Med Care 1992;30:473-83.

24. Farivar SS, Cunningham WE, Hays RD. Correlated physical and mental health summary scores for the SF-36 and SF-12 Health Survey, V.I. Health Qual Life Outcomes 2007;5:54.

25. Fritzmaurice GM, Laird NM, Ware JH. Applied longitudinal analysis. 2nd edn. Hoboken, NJ: Wiley, 2011.

26. Cohen J, Cohen P, West SG, et al. Applied multiple regression/ correlation analysis for the behavioral sciences. 3rd edn. London: Lawrence Erlbaum Associates, 2003.

27. Wiebe S, Guyatt G, Weaver B, et al. Comparative responsiveness of generic and specific quality-of-life instruments. J Clin Epidemiol 2003;56:52-60.

28. Sampat S, Kirschenbaum DS, Gierut KJ, et al. Ya gotta have friends: social support and self-efficacy predict success following immersion treatment. Obesity 2014;22:n/a-2585

29. Finne E, Reinehr T, Schaefer A, et al. Changes in self-reported and parent-reported health-related quality of life in overweight children and adolescents participating in an outpatient training: findings from a 12-month follow-up study. Health Qual Life Outcomes 2013;11:1.

30. Warschburger P, Fromme C, Petermann F. Gewichtsbezogene Lebensqualität bei Schulkindern: Validität des GW-LQ-KJ. Zeitschrift für Gesundheitspsychologie 2004;12:159-66.

31. Wong WW, Barlow SE, Mikhail C, et al. A residential summer camp can reduce body fat and improve health-related quality of life in obese children. J Pediatr Gastroenterol Nutr 2013;56:83-5.

32. Hoedjes M, Makkes S, Halberstadt J, et al. Health-related quality of life in children and adolescents with severe obesity after intensive lifestyle treatment and at 1-year follow-up. Obes Facts 2018;11:116-28.

33. Kolotkin RL, Zeller M, Modi AC, et al. Assessing weight-related quality of life in adolescents. Obesity 2006;14:448-57.

34. Rank M, Wilks DC, Foley L, et al. Health-related quality of life and physical activity in children and adolescents 2 years after an inpatient weight-loss program. J Pediatr 2014;165:732-7.

35. Erhart M, Ellert U, Kurth BM, et al. Measuring adolescents' HRQoL via self reports and parent proxy reports: an evaluation of the psychometric properties of both versions of the KINDL-R instrument. Health Qual Life Outcomes 2009;7:77. 